\title{
Clinical characteristics and prognosis of ovarian clear cell carcinoma: a 10-year retrospective study
}

Chenchen Zhu ${ }^{1 \dagger}$, Jing Zhư ${ }^{2 \dagger}$, Lili Qian², Hanyuan Liu², Zhen Shen², Dabao Wu², Weidong Zhao ${ }^{2}$, Weihua Xiao ${ }^{3,4}$ and Ying Zhou ${ }^{1,2^{*}}$ (i)

\begin{abstract}
Background: Ovarian clear cell carcinoma (OCCC) is a special pathological type of epithelial ovarian carcinoma (EOC). We conducted this research to investigate the clinical characteristics and outcomes of OCCC and to provide additional supporting evidence to aid in the clinical diagnosis and management.

Methods: This was a retrospective study investigating the clinical characteristics and survival outcomes of 86 patients with OCCC treated at our center between January 2010 and March 2020. Survival analysis was also performed on 179 patients with OCCC obtained from the Surveillance, Epidemiology and End Results (SEER) cancer registry database.

Results: The median age of participants was $49.21 \pm 9.91$ years old, and $74.42 \%$ of them were diagnosed at early stage. The median CA125 level was $601.48 \mathrm{IU} / \mathrm{mL}$, while $19.77 \%$ of the patients had normal CA125 levels. Sixteen patients (18.60\%) had co-existing endometriosis and 8 patients (9.3\%) developed venous thromboembolism (VTE). There were 5 patients received suboptimal cytoreduction. Sixty-six patients (76.74\%) underwent lymphadenectomy, and only 3 (4.55\%) patients had positive lymph nodes. Patients diagnosed at an early stage had higher 3-year overall survival (OS) and progression-free survival (PFS) rates than those with advanced stage OCCC. CA19-9 $(P=$ $0.025)$ and ascites $(P=0.001)$ were significantly associated with OS, while HE4 $(P=0.027)$ and ascites $(P=0.001)$ were significantly associated with PFS. Analysis of data from the SEER database showed that positive lymph nodes is also an independent prognostic factor for OS $(P=0.001)$.
\end{abstract}

Conclusions: OCCC often presents at an early stage and young age with a mildly elevated CA125. CA19-9, HE4, massive ascites, and positive lymph node are independent prognostic factors.

Keywords: Ovarian clear cell carcinoma, Biomarker, Survival, Prognostic factor

\footnotetext{
* Correspondence: caddiezy@ustc.edu.cn

${ }^{+}$Chenchen Zhu, and Jing Zhu are co-first author

'Department of Obstetrics and Gynecology, Anhui Provincial Hospital, Anhui

Medical University, Hefei 230001, China

2Department of Obstetrics and Gynecology, The First Affiliated Hospital of

USTC, Division of Life Sciences and Medicine, University of Science and

Technology of China, Hefei 230001, Anhui, China

Full list of author information is available at the end of the article
}

(C) The Author(s). 2021 Open Access This article is licensed under a Creative Commons Attribution 4.0 International License, which permits use, sharing, adaptation, distribution and reproduction in any medium or format, as long as you give appropriate credit to the original author(s) and the source, provide a link to the Creative Commons licence, and indicate if changes were made. The images or other third party material in this article are included in the article's Creative Commons licence, unless indicated otherwise in a credit line to the material. If material is not included in the article's Creative Commons licence and your intended use is not permitted by statutory regulation or exceeds the permitted use, you will need to obtain permission directly from the copyright holder. To view a copy of this licence, visit http://creativecommons.org/licenses/by/4.0/ The Creative Commons Public Domain Dedication waiver (http://creativecommons.org/publicdomain/zero/1.0/) applies to the data made available in this article, unless otherwise stated in a credit line to the data. 


\section{Background}

Epithelial ovarian carcinoma (EOC) is the seventh most commonly diagnosed cancer among women worldwide, and carries the highest mortality rate of all gynecological cancers $[1,2]$. Ovarian clear cell carcinoma (OCCC) is a subtype of EOC with differing prevalence depending on geographical location. OCCC accounts for 5-10\% of all EOC in North America and $12 \%$ in western countries, but appears to have a higher prevalence in East Asia, accounting for $25-30 \%$ and $10.3-11.6 \%$ of all EOC in Japan and Korea, respectively [3-5].

There are several reproductive and hormonal risk factors linked to an increased risk of developing OCCC, such as early menarche, late menopause, low use of oral contraceptives, and low pregnancy rate [6]. In addition, endometriosis is recognized as a precancerous lesion of OCCC, as women with endometriosis have a 3-fold increased risk of developing OCCC compared with women without endometriosis [7]. Patients with OCCC tend to be diagnosed at younger age and earlier stage, and occasionally are found to have thromboembolic complications [8], usually with a mild-to-moderate elevation of serum CA125. The conventional tumor marker CA125 used for detection of high-grade serous carcinoma (HGSC) is a poor marker for OCCC, elevated in only $57.6 \%$ of OCCC cases with a high false-negative rate [9]. Therefore, there is a need for specific serological biomarkers for OCCC. Immunohistochemically, OCCC is usually positive for hepatocyte nuclear factor $1 \beta$ $(\mathrm{HNF} 1 \beta)$ and negative for estrogen receptor (ER), progesterone receptor (PR), and Wilms Tumor 1 (WT-1) in more than $95 \%$ of the cases [10]. OCCC shows little association with family history, and BRCA1 and BRCA2 germline mutations are rare in OCCC, however, somatic mutations of phosphatidylinositol-4,5-bisphosphate 3kinase catalytic subunit alpha (PIK3CA) and AT-rich interactive domain-containing protein 1A (ARID1A) are present in $20-51 \%$ and $40-57 \%$ respectively $[11,12]$.

Standard surgical staging procedure or optimal cytoreduction, followed by systemic chemotherapy, is recommended as the primary treatment for patients with OCCC. However, the response rate of platinum-based chemotherapy is only 20 to $50 \%$ for OCCC, therefore there is a need for further research into more effective therapies [13]. Due to this inherent chemoresistance, the prognosis of patients with OCCC is extremely poor, especially at advanced stage. Suboptimal cytoreduction, lymph node (LN) metastasis, and occurrence of VTE are also prognostic predictors of poor outcome [13, 14].

Therefore, the purpose of our research is to assess the clinical characteristics and outcomes of patients with OCCC, and to provide additional supporting evidence to aid in the clinical diagnosis and management of OCCC.

\section{Materials and methods}

\section{Patients}

This is a retrospective study of 86 patients diagnosed with primary ovarian clear cell carcinoma between January 2010 and March 2020 at The First Affiliated Hospital of University of Science and Technology of China (USTC). Patients with histologically confirmed OCCC with only pure clear cell histology by pathologists who had undergone complete surgical staging or cytoreductive surgery with adjuvant chemotherapy as the primary treatment were included. Patients were excluded from this study if they received neoadjuvant chemotherapy, had insufficient data, or were lost to follow-up within 1 month of surgery. Patient information, including demographic and pathological characteristics, pre-operative biomarkers, surgical procedure, chemotherapy, and disease status at last contact, was collected from medical records and evaluated. Patient records and information were anonymized prior to analysis; thus, consent was not required. This study was approved by the ethics committees of The First Affiliated Hospital of USTC and was conducted in accordance with the Helsinki Declaration.

\section{Treatment and follow-up}

The predominant primary surgical procedure was total hysterectomy, bilateral salpingo-oophorectomy, lymphadenectomy, and omentectomy, with peritoneal biopsies from multiple random sites. Twenty-five patients with a pelvic mass had previously undergone surgical procedures including oophorocystectomy, unilateral/bilateral ovariosalpingectomy \pm total hysterectomy, and were diagnosed with OCCC according to the pathological results from our or other hospitals and subsequently received additional staging or cytoreductive surgery in our hospital. The other 61 patients were diagnosed and treated within our hospital. Firstline adjuvant chemotherapy was combined platinum and taxanes of 3-6 cycles.

After the initial treatment, all patients were closely followed up with clinical examination, including pelvic examination and evaluation of tumor markers at each visit. In addition, ultrasound, computed tomography (CT), magnetic resonance imaging (MRI), and/or positron emission tomography-CT (PET-CT) scans were performed when necessary. Where follow-up information was not available from patient records, patients were contacted directly by telephone to obtain the relevant information. Recurrence was defined as histologic evidence by tumor biopsy or fine-needle biopsy and/or the appearance of new lesions on imaging. Survival data were last collected on 31 April 2020. 


\section{Clinical data collection}

The following information was collected from the medical records of eligible patients: age, body mass index (BMI), results of genetic tests, presence of endometriosis, history of thromboembolism, stage, comorbidities, American Society of Anesthesiologists class (ASA), stage, preoperative serum laboratory test values, surgical procedures performed, presence of ascites, size of residual tumor, number of LN removed, presence of LN metastasis, pathologic results, length of hospital stay, chemotherapy regimen, length of follow-up, recurrence and survival status.

Fasting venous blood samples were collected from all patients on the morning prior to their planned surgery. Electrochemiluminescence immunoassay (ELICA) was performed on all samples using the Cobas E601 analyzer (Roche Diagnostics) to measure the levels of CA125, HE4, and CA19-9 For patients who had undergone primary surgery at other institutes, the results of serum analysis were collected from their medical notes. All tumors were staged according to the 2014 International Federation of Gynecology and Obstetrics (FIGO) staging system. In patients treated prior to 2014, the stage of disease was classified retrospectively on the basis of surgical and pathological assessment. Optimal cytoreduction was defined as the maximal diameter of the residual tumor $\leq 1 \mathrm{~cm}$ following surgery. Progression-free survival (PFS) was defined as the time from initial surgical staging or cytoreductive surgery to the date of disease progression or recurrence, and overall survival (OS) was defined as the time from surgical staging or cytoreductive surgery to the date of death, or to the last follow-up date, if still alive.

The histological cell types were determined according to the World Health Organization (WHO) criteria, and the diagnosis was conducted by at least two pathologists. Pathological slides of patients who underwent primary surgery at other institutes were obtained for histological reconfirmation. The presence of endometriosis was obtained from pathological reports.

\section{Data collection from the surveillance, epidemiology and end results (SEER) database}

Patients' data were collected from the latest version of the SEER cancer registry database. There were 849 cases initially identified with a diagnosis of OCCC from 1975 to 2017 with at least 3 years of follow-up available. Exclusion criteria were as follows: incomplete clinical information (621 cases), no surgical procedure performed (8 cases), and the presence of primary malignancy elsewhere (41 cases). Following the selection process, a total of 179 eligible patients were enrolled in the study. Survival analysis was performed using the following demographic and clinicopathological parameters: age, race (white, black, or 'other'), SEER summary stage (localized, regional, or distant), American Joint Committee on Cancer (AJCC) stage (I, II, III, or IV), number of LN resected $(1,2,3$, or $\geq 4)$, number of positive $\operatorname{LN}(1,2,3$, or $\geq 4)$, and the presence of distant metastasis.

\section{Statistic analysis}

Statistical analysis was performed using SPSS v.20.0 software (IBM Corp., Armonk, NY, USA). We conducted the power calculation using PASS v11.0 software. Continuous variables were expressed as mean, standard deviation, and range, and categorical variables were expressed as counts and percentages. We constructed the reverse Kaplan-Meier curve by reversing the "censor" and "event" of the standard Kaplan-Meier curve. Comparisons between groups were analyzed using Student's t-test or Wilcoxon-Mann-Whitney test according to the data distribution for continuous variables or the $\chi^{2}$ or Fisher's exact test for categorical variables. Univariate and multivariable Cox regression analyses were performed to identify predictors of RFS and OS. Multivariate analysis was performed with all variables with a $p$ value of $<0.1$ at univariate analysis. Multivariate logistic regression analysis was also performed with bootstrap in SPSS. A $p$-value of $<0.05$ was considered statistically significant, and all $p$-values reported were two-sided.

\section{Results}

The characteristics of the patients with OCCC

Overall, 86 patients were included in this study who were diagnosed with OCCC between January 2010 and March 2020. The demographic and clinical characteristics of these patients are summarized in Table 1. Twenty-five patients with a pelvic mass had previously undergone surgical procedures including oophorocystectomy, unilateral/bilateral ovariosalpingectomy \pm total hysterectomy, and were diagnosed with OCCC in our or other hospitals according to pathological results. Of these patients, 24 of them received additional staging surgery and were diagnosed as early stage, and one patient received cytoreductive surgery and was diagnosed as FIGO IIIC in our hospital. The other 61 patients received primary cytoreductive or staging surgery within our hospital, and detailed information is presented in Fig. 1.

The median age at diagnosis was $49.21 \pm 9.91$ years (range 25-70 years). The majority of patients (64/86, $74.42 \%)$ were diagnosed as early-stage (FIGO IA-IIIB). In terms of gene analysis, only $6.67 \%(1 / 15)$ displayed a positive BRCA mutation. CA125 assay was performed in 71 patients, with a median CA125 level of $601.48 \mathrm{IU} / \mathrm{mL}$ (range $9.16-9035 \mathrm{IU} / \mathrm{mL}$ ). Of these 71 patients, 17 (19.77\%) had a normal CA125 level ( mean $=20.21 \pm 7.72$ $\mathrm{U} / \mathrm{mL})$. More than half of patients assayed had a normal 
Table 1 Baseline clinical characteristics of patients $(n=86)$

Age (years)
$<40$
$40 \sim 49$
$50 \sim 59$
$\geq 60$
Body mass index $\left(\mathbf{k g} / \mathbf{m}^{2}\right)$
$<18$
$18 \sim 23.9$
$24 \sim 27.9$
$\geq 28$
NA

$49.21 \pm 9.91(25 \sim 70)$

15

27

$22.96 \pm 3.01(16.94 \sim 33.71)$

2

26

3

FIGO stage

।

IA

$\mathrm{IB}$

IC

IC1

IC2

IC3

IC NA

II

IIA

IIB

III

IIIA

IIIB

IIIC

IV

BRCA mutation ( $n=15)$

Unknown significance mutation

\section{Endometriosis}

Thrombosis

Preoperative

postoperative

$\mathrm{CDC}^{\mathrm{b}}$

Hypertension
Diabetes
Heart disease
Cerebral infarction
Other cancer history
Hepatic cancer
Breast cancer
Cervical cancer
Endometrial cancer
Hepatitis
Systemic lupus erythematosus
Rheumatoid arthritis
Bronchial asthma


Table 1 Baseline clinical characteristics of patients $(n=86)$ (Continued)

\begin{tabular}{lll}
\hline & No & $\%$ \\
\hline Hypothyroidism & 1 & 1.16 \\
Moyamoya disease & 1 & 1.16 \\
Tuberculosis & 1 & 1.16 \\
American Society of Anesthesiologists class (ASA) & & \\
I & 7 & 8.14 \\
II & 39 & 45.35 \\
III & 31 & 36.05 \\
IV & 3 & 3.49 \\
NA & 6 & 6.98
\end{tabular}

\section{Preoperative laboratory test}

CA125(U/ml) $)^{a}$
Normal $(<35)$
$35 \sim 99$
$100 \sim 499$
$500 \sim 999$
$\geq 1000$
NA
CA19-9(U/ml) ${ }^{\text {a }}$
Normal $(<37)$
$37 \sim 99$
$100 \sim 499$
$\geq 500$

NA

$601.48 \pm 1546.66(9.16 \sim 9035.00)$

17

$1575.97 \pm 11,885.76(0.60 \sim 96,649.00)$

HE4 (pM) ${ }^{\text {a }}$

Normal $(<140)$

$105.37 \pm 102.93(14.00 \sim 669.00)$

$140 \sim 499$

$<40$

$\geq 40$

NA

Prealbumin (mg/l) ${ }^{\text {a }}$

$194.65 \pm 56.29(58.00 \sim 332.00)$

$<170$

D-Dimer (ug/ml) ${ }^{\text {a }}$

$2.21 \pm 3.19(0.03 \sim 12.86)$

$<3.5$

$\geq 3.5$

$\mathrm{Ca}^{2+}(\mathrm{mmol} / \mathrm{l})^{\mathrm{a}}$ 


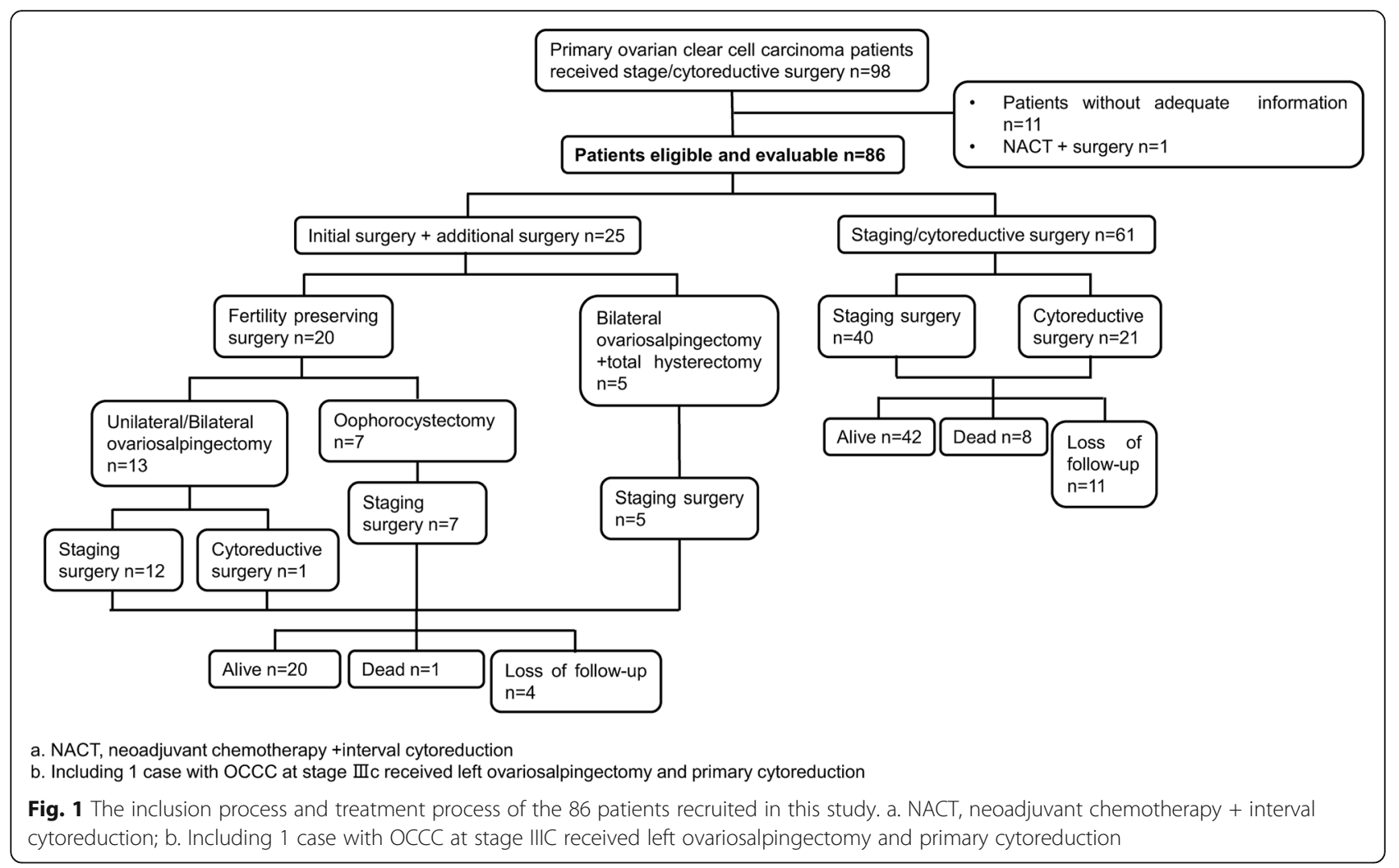

CA19-9 $($ mean $=12.96 \pm 9.01 \mathrm{U} / \mathrm{mL})$ and $/$ or HE4 value $(-$ mean $=65.86 \pm 35.70 \mathrm{pM})(36 / 66,54.55 \% ; 45 / 59,76.27 \%)$ respectively. Co-existing endometriosis was found in 16 patients $(18.60 \%)$ and 8 patients $(9.30 \%)$ had a preoperative history or postoperative complications of VTE (Table 2).

\section{Treatments of the patients with OCCC}

Complete staging surgery was performed in 64 (74.42\%) patients and cytoreductive surgery was performed in 22 (25.58\%) patients. Data related to the surgical procedures are summarized in Table 3. Lymphadenectomy was performed in 66 women (76.74\%), and three of them (4.55\%) were found to have positive LN. Of the $2225 \mathrm{LN}$ resected, $2.07 \%(46 / 2225)$ were found to have metastatic lesions. $85.94 \%(55 / 64)$ patients at early stage had over 20 lymph nodes removed), whereas $77.27 \%$ (17/22) patients at advanced stage had less than 20 lymph nodes removed. Among patients with advanced OCCC, optimal cytoreduction was achieved in $77.27 \%(17 / 22)$ patients. Of the 5 patients that underwent suboptimal cytoreductive surgery, 1 patient's family refused to remove the involved bowel, and 4 patients had multiple metastatic lesions. The mean length of hospital stays for patients undergoing staging surgery was $11.44 \pm 6.45$ days following completion of initial postoperative chemotherapy; and the mean length of hospital stays for those undergoing cytoreductive surgery was $15.00 \pm 6.50$ days following completion of initial postoperative chemotherapy.

Adjuvant chemotherapy was administered in our hospital in 76 patients $(88.37 \%)$, with a combination of paclitaxel and platinum following surgery. The other 10 patients were discharged and attended a local hospital for their adjuvant chemotherapy. Among the 76 patients who received chemotherapy in our hospital, $36(47.37 \%)$ completed the initial chemotherapy within 2 weeks of surgery and 68 $(89.47 \%)$ within 4 weeks of surgery. The remaining 8 patients did not complete chemotherapy within 4 weeks due to anemia, infection, or personal reasons. The mean interval between surgery and initial postoperative chemotherapy was $16.76 \pm 8.01$ days (range 639 days).

\section{Clinical characteristics of OCCC patients with co-existing endometriosis}

There were 16 patients found to have co-existing endometriosis. A comparison of the clinical characteristics of patients with and without co-existing endometriosis is presented in sTable1. Patients with coexisting endometriosis had a mean age of $45.50 \pm 6.19$ years old, and $68.75 \%$ of them were under 50 years old. Patients with endometriosis tended to be younger than those without endometriosis but without statistical significance $(P=0.078)$. 
Table 2 Characteristic of patients with developed venous thromboembolism (VTE)

\begin{tabular}{|c|c|c|c|c|c|c|c|c|c|c|c|c|c|c|}
\hline Case & Age & FIGO stage & Ascites & SCS & Ro/R1/RX & $D-D^{a}$ & $D-D^{b}$ & CA125 & CA19-9 & Time & DVT & $\mathrm{PE}$ & Survival & OS \\
\hline 1 & 55.00 & IIIC & 3000 & 2 & RO & 1.36 & 15.32 & 1664 & 96,649 & Pre-operation & Left lower extremity & & Alive & 9 \\
\hline 2 & 42.00 & IIIC & 2000 & 4 & RO & 12.86 & 17.42 & 446.2 & 35.78 & Pre-operation & - & Yes & Alive & 24 \\
\hline 3 & 45.00 & IC & 200 & 4 & RO & / & 13.68 & 2612 & 10.72 & Pre-operation & Right lower extremity & & Alive & 75 \\
\hline 4 & 40.00 & $\| I C$ & 200 & 2 & R1 & 8.77 & 40 & 239.3 & 251.7 & Pre-operation & Both lower extremity & Yes & Alive & 24 \\
\hline 5 & 70.00 & IV & 0 & 1 & RX & 2.43 & 5.25 & 911.5 & 1469 & Post-operation & - & Yes & Alive & 10 \\
\hline 6 & 25.00 & IV & 2000 & 1 & RO & 12.48 & 5.69 & 9035 & 2.96 & Post-operation & Both lower extremity & & NA & - \\
\hline 7 & 66.00 & $\mid A$ & 0 & 5 & RO & 0.78 & 6.15 & 9.5 & 10.54 & Post-operation & Both lower extremity & Yes & Alive & 9 \\
\hline 8 & 56.00 & IA & 0 & 3 & RO & / & / & 103.1 & 8.42 & Post-operation & Both lower extremity & & Alive & 13 \\
\hline
\end{tabular}

SCS Surgical complexity score

DVT Deep venous thrombosis

$P E$ Pulmonary embolism

OS Overall survival

apreoperative d-dimer value

${ }^{b}$ Postoperative d-dimer value

\section{Pathological characteristics}

The immunohistochemical results are presented in Table 4. Most (26/31, 83.87\%) patients tested positive for HNF1 $\beta$ and $91.67 \%$ (55/60), 71.93\% (41/57), and $82.00 \%(41 / 50)$ of patients tested negative for WT-1, ER, and PR respectively. Of the 20 patients who were tested containing HNF1 $\beta$, WT-1, ER, and PR, 11 (55\%) had the combination of positive HNF1 $\beta$, and negative WT-1, ER, and PR characteristics for the diagnosis of clear cell carcinoma. Of these 20 patients, 19 were alive and one was lost to follow-up. In addition, Ki-67 was positive in 1$20 \%$ of cells in $18 / 69(26.09 \%)$ cases; $21-40 \%$ of cells in $26 / 69$ (37.68\%) cases; $41-60 \%$ of cells in $17 / 69$ (24.64\%) cases; $61-80 \%$ of cells in 7/69 (10.14\%) cases; and > 80\% of cells in $1 / 69$ (1.45\%) cases.

\section{Survival analysis of the clinical data}

The median follow-up of the cohort (86 patients) was 53 months based on the reverse Kaplan-Meier method. Among the cohort, 14 patients experienced relapse shown in sTable2. After recurrence, five cases were performed with surgery and received chemotherapy, three of them are still alive, and the median post-relapse survival is 13 months; while eight cases were only performed with chemotherapy after recurrence and two cases are still alive, and the median post-relapse survival is 11 months. Among the seven patients having complete information about the chemotherapy after recurrence, one patient received ifosfamide + etoposide, and all the other six patients received taxanes \pm platinum for relapse management. And the median post-relapse survival of these 14 patients is 13 months based on Kaplan-Meier analysis.

There were $71(81.61 \%)$ patients follow-up continuously. Among the 71 patients followed up, 49 were followed up for more than 1 year, 28 for more than 3 years, and only 7 for more than 5 years. Survival analysis was conducted among the 49 patients followed up for more than 1 year. Recurrence occurred in 13 patients (26.53\%), including 7 cases at an advanced stage and 6 cases at an early stage. For patients with early and advanced stage OCCC, 1-year PFS rates were 94.59 and $33.33 \%$ respectively and 3-year PFS rates were 78.95 and $22.22 \%$ respectively; OS rates at 1-year were 97.30 and $66.67 \%$ respectively and at 3 -years were 89.47 and $44.44 \%$ respectively. We also compared the median survival time of patients with IC1 or IC $2 / 3$ of OCCC, and the median PFS and OS of them are shown in sTable3 and sTable4. Although the statistical significance was not calculated due to the small number of cases, it can be clearly found that the prognosis of patients at stage IC1 (49 months) is better than that at IC2/3 (37.6 months).

Analysis of demographic and clinicopathological parameters associated with OS and PFS is presented in Table 5. Univariate analysis demonstrated that CA19$9 \geq 70.3 \mathrm{U} / \mathrm{mL} \quad(P=0.038)$, advanced stage $(P=0.001)$ and ascites $\geq 2000 \mathrm{~mL}(P=0.015)$ were significantly associated with reduced OS. Besides those factors, suboptimal cytoreduction $(P=0.085)$ was also included in multivariate analysis. Multivariate analysis revealed that CA19-9 $\geq 70.3 \mathrm{U} / \mathrm{mL}(P=0.025)$ was an independent prognostic indicator of OS. Bootstrap analysis found ascites $\geq 2000 \mathrm{~mL} \quad(P=0.001) \quad$ also had statistical significance.

In univariate analyses of PFS, age $\geq 58$ years $(P=0.038)$ and advanced stage $(P=0.002)$ were significantly associated with reduced PFS. Age $\geq 58$ years and advanced stage, along with CA19-9 $\geq 70.3 \mathrm{U} / \mathrm{mL}, \mathrm{HE} 4 \geq 94.5 \mathrm{pM}$, and ascites $\geq 2000 \mathrm{~mL}$ were included in multivariate analysis. None of these factors had statistical significance, however, the additional bootstrap analysis found that HE $4 \geq 94.5 \mathrm{pM} \quad(P=0.027)$ and ascites $\geq 2000 \mathrm{~mL}(P=$ $0.001)$ were associated with a decreased PFS. 
Table 3 Surgical procedures and clinical outcomes

\begin{tabular}{|c|c|c|c|c|c|c|}
\hline \multirow{3}{*}{ Residual disease } & \multirow[t]{2}{*}{ Total } & \multirow[t]{2}{*}{$\%$} & \multicolumn{4}{|l|}{ FIGO stage } \\
\hline & & & \multirow[t]{2}{*}{$\mathrm{I} \sim \mathrm{IIIb}(n=64)$} & \multirow[t]{2}{*}{$\%$} & \multirow[t]{2}{*}{ IIIC $\sim \operatorname{IV}(n=22)$} & \multirow[t]{2}{*}{$\%$} \\
\hline & & & & & & \\
\hline & 77 & 89.53 & 64 & 100 & 13 & 59.09 \\
\hline $0.1 \sim 1.0 \mathrm{~cm}$ & 4 & 4.65 & 0 & 0 & 4 & 18.18 \\
\hline$>1 \mathrm{~cm}$ & 5 & 5.81 & 0 & 0 & 5 & 22.73 \\
\hline \multicolumn{7}{|l|}{ Ascites (ml) } \\
\hline$<500$ & 69 & 80.23 & 59 & 92.19 & 10 & 45.45 \\
\hline $500 \sim 1999$ & 7 & 8.14 & 3 & 4.69 & 4 & 18.18 \\
\hline $2000 \sim 4999$ & 5 & 5.82 & 0 & 0 & 5 & 22.73 \\
\hline$\geq 5000$ & 3 & 3.49 & 0 & 0 & 3 & 13.64 \\
\hline NA & 2 & 2.33 & 2 & 3.13 & 0 & \\
\hline \multicolumn{7}{|l|}{ Number of lymph node resected } \\
\hline 0 & 20 & 23.26 & 5 & 7.81 & 15 & 68.18 \\
\hline $1 \sim 20$ & 6 & 6.98 & 4 & 6.25 & 2 & 9.09 \\
\hline $21 \sim 40$ & 45 & 52.33 & 41 & 64.06 & 4 & 18.18 \\
\hline$\geq 40$ & 15 & 17.44 & 14 & 21.88 & 1 & 4.55 \\
\hline Lymph node ratio (LNR) ${ }^{a}$ & $46 / 2225$ & 2.07 & $45 / 2034$ & 2.21 & $1 / 191$ & 0.52 \\
\hline \multicolumn{7}{|l|}{ Estimated blood loss (ml) } \\
\hline$<100$ & 4 & 4.65 & 3 & 4.69 & 1 & 4.55 \\
\hline $100 \sim 499$ & 46 & 53.49 & 38 & 59.38 & 8 & 36.36 \\
\hline $500 \sim 999$ & 26 & 30.23 & 19 & 29.69 & 7 & 31.82 \\
\hline$\geq 1000$ & 9 & 10.47 & 3 & 4.69 & 6 & 27.27 \\
\hline NA & 1 & 1.16 & 1 & 1.56 & 0 & 0 \\
\hline \multicolumn{7}{|l|}{ Operation type } \\
\hline Staging surgery & 64 & 74.42 & 64 & 100 & 0 & 0 \\
\hline Standard cytoreduction & 13 & 15.12 & 0 & 0 & 13 & 59.09 \\
\hline Radical cytoreduction & 1 & 1.16 & 0 & 0 & 1 & 4.55 \\
\hline Extral-radical cytoreduction & 3 & 3.49 & 0 & 0 & 3 & 13.64 \\
\hline Palliative surgery & 5 & 5.81 & 0 & 0 & 5 & 22.73 \\
\hline \multicolumn{7}{|l|}{ Surgical complexity score (SCS) } \\
\hline $1 \sim 3$ & 26 & 30.23 & 14 & 21.88 & 12 & 54.55 \\
\hline $4 \sim 7$ & 59 & 68.60 & 50 & 78.13 & 9 & 40.91 \\
\hline$\geq 8$ & 1 & 1.16 & 0 & 0 & 1 & 4.55 \\
\hline Interval of initial postoperative chemotherapy (days) & \multicolumn{2}{|c|}{$16.76 \pm 8.01(6 \sim 39)$} & \multicolumn{2}{|c|}{$17.86 \pm 7.94(6 \sim 39)$} & \multicolumn{2}{|c|}{$13.47 \pm 7.28(6 \sim 32)$} \\
\hline Length of hospital stays (days) & \multicolumn{2}{|c|}{$12.15 \pm 6.54(5 \sim 37)$} & \multicolumn{2}{|c|}{$11.44 \pm 6.45(5 \sim 37)$} & \multicolumn{2}{|c|}{$15.00 \pm 6.50(8 \sim 35)$} \\
\hline Recurrence & 13 & 15.12 & 6 & 9.38 & 7 & 31.82 \\
\hline \multicolumn{7}{|l|}{ Endpoint status } \\
\hline Alive & 62 & 72.09 & 52 & 81.25 & 10 & 45.45 \\
\hline Cancer specific deaths & 10 & 11.63 & 2 & 3.13 & 8 & 36.36 \\
\hline Loss to follow-up & 14 & 16.28 & 10 & 15.63 & 4 & 18.18 \\
\hline
\end{tabular}

aLymph node ratio (LNR), defined as the ratio of the number of metastatic lymph nodes (MLNs) to the number of resected lymph nodes (RLNs)

We have also calculated the power regarding the ability to detect significant differences of survival analysis and the multiple hypothesis correction using False
Discovery Rate (FDR) approach for the indicators included in the multivariate analysis among these 49 patients, the power and adjusted $p$ values were presented 
Table 4 Immunohistochemical characteristics of patients

\begin{tabular}{lllll}
\hline & Total number $^{\mathbf{a}}$ & Positive (\%) & Negative (\%) & Weak/partial positive (\%) \\
\hline HNF13 & 31 & $26 / 31(83.87 \%)$ & $1 / 31(3.23 \%)$ & $4 / 31(12.90 \%)$ \\
WT-1 & 60 & $2 / 60(3.33 \%)$ & $55 / 60(91.67 \%)$ & $3 / 60(5.00 \%)$ \\
ER & 57 & $3 / 57(5.26 \%)$ & $41 / 57(71.93 \%)$ & $13 / 57(22.81 \%)$ \\
PR & 50 & $1 / 50(2.00 \%)$ & $41 / 50(82.00 \%)$ & $8 / 50(16.00 \%)$ \\
NapsinA & 60 & $29 / 60(48.33 \%)$ & $22 / 60(36.67 \%)$ & $9 / 60(15.00 \%)$ \\
CK7 & 66 & $61 / 66(92.42 \%)$ & $3 / 66(4.55 \%)$ & $2 / 66(3.03 \%)$ \\
P53 & 54 & $16 / 54(29.63 \%)$ & $16 / 54(29.63 \%)$ & $22 / 54(40.74 \%)$ \\
P16 & 25 & $13 / 25(52.00 \%)$ & $4 / 25(16.00 \%)$ & $8 / 25(32.00 \%)$ \\
PAX8 & 41 & $40 / 41(97.56 \%)$ & $1 / 41(2.44 \%)$ & 0 \\
CK20 & 0 & $32 / 34(94.12 \%)$ & $2 / 34(5.88 \%)$ \\
CD10 & 34 & $1 / 18(5.56 \%)$ & $14 / 18(77.78 \%)$ & $3 / 18(16.67 \%)$ \\
CD15 & 18 & $24 / 47(51.06 \%)$ & $11 / 47(23.40 \%)$ & $12 / 47(25.53 \%)$ \\
Vim & 47 & $7 / 38(18.42 \%)$ & $28 / 38(73.68 \%)$ & $3 / 38(7.89 \%)$ \\
CA125 & 38 & $26 / 37(70.27 \%)$ & $4 / 37(10.81 \%)$ & $7 / 37(18.92 \%)$ \\
\hline
\end{tabular}

${ }^{\mathrm{a}}$ Number of patients who had this test

in sTable7 and sTable8. Unfortunately, none of the adjusted $p$ values is statistically significant, which might be caused by the small samples of the patients in this study. Thus, a large-sample multicentral clinical research is urgently needed.

\section{Analysis of data from the SEER database}

We analyzed the data of 179 patients enrolled from the SEER database, with an average age of $55.22 \pm 10.47$ (range 18-85) years. In terms of SEER summary stage, there were 55 cases of localized, 77 cases of regional and 47 cases of distant, while 40 patients were diagnosed at advanced stage (AJCC IIIC and IV). Lymphadenectomy was performed in 134 patients, of which 19 (14.18\%) had positive LN. We also found that 6 patients had distant metastasis in bone ( 1 case), lung (1 case), and liver (4 cases).

Survival analysis was performed on these 179 cases of OCCC with a 3-year OS rate of 56.98\%. Kaplan-Meier survival curves (Fig. 2) were generated which showed that AJCC stage $(P<0.001)$, SEER summary stage $(P<$ $0.001), \geq 4 \mathrm{LN}$ removed $(P=0.003)$, and positive $\mathrm{LN}$ $(P<0.001)$ were significantly associated OS in patients with OCCC. Multivariate analysis was performed with variables including AJCC stage, SEER summary stage, number of LN removed, and positive LN (Table 6). Patients with summary stage of distant had poorer survival $(P=0.011)$. Positive $\mathrm{LN}$ was an independent prognostic factor for the survival of OCCC $(P=0.001)$. AJCC stage and number of $\mathrm{LN}$ removed had no significant impact on OS.

\section{Discussion}

OCCC is a rare pathological type of EOC, and there is geographic variance in the prevalence of OCCC, being more common in Asia [3-5]. Prevalence also differs by race, being higher in Asians (11.1\%) and lower in black, white, and other populations $(3.1,4.8$, and $5.5 \%$, respectively) [15]. However, among patients analyzed from the SEER database in our research, only $15.08 \%$ of the 179 cases were Asians, with $78.21 \%$ of cases being white; this is likely related to the racial differences in the USA population. In the present study, $74.42 \%$ patients were diagnosed at an early stage in a younger age (49.21 \pm 9.91), consistent with previous studies, showing a distinct epidemiology of OCCC from HGSC, which is more frequently diagnosed at an advanced stage with a poor prognosis [8].

Endometriosis is a common disease in women of reproductive age, which is recognized as a precancerous lesion of OCCC and is associated with triple the risk of OCCC $[7,16]$, approximately $18-43 \%$ of women with OCCC have a history of endometriosis $[17,18]$. Endometriotic lesions often have high expression of HNF1 $\beta$ and carry multiple somatic mutations, such as ARID1A and PIK3CA, which are thought to occur early in the malignant transformation of OCCC [16]. The risk of tumorigenesis in endometriosis is about $1 \%$ among premenopausal women and $1-2.5 \%$ among postmenopausal women $[17,19]$. A study by Ye et al. demonstrated that patients with OCCC and concurrent endometriosis were on average 8 years younger than those without, and were more likely to present at early stage (78.5\%) [20]. However, although patients with co-existing endometriosis tend to have better survival outcomes, endometriosis was not an independent predictor of survival [21]. In the present study, OCCC patients with endometriosis also tended to be diagnosed at a younger age (average age of $45.50 \pm 6.19) \quad$ compared to patients without 


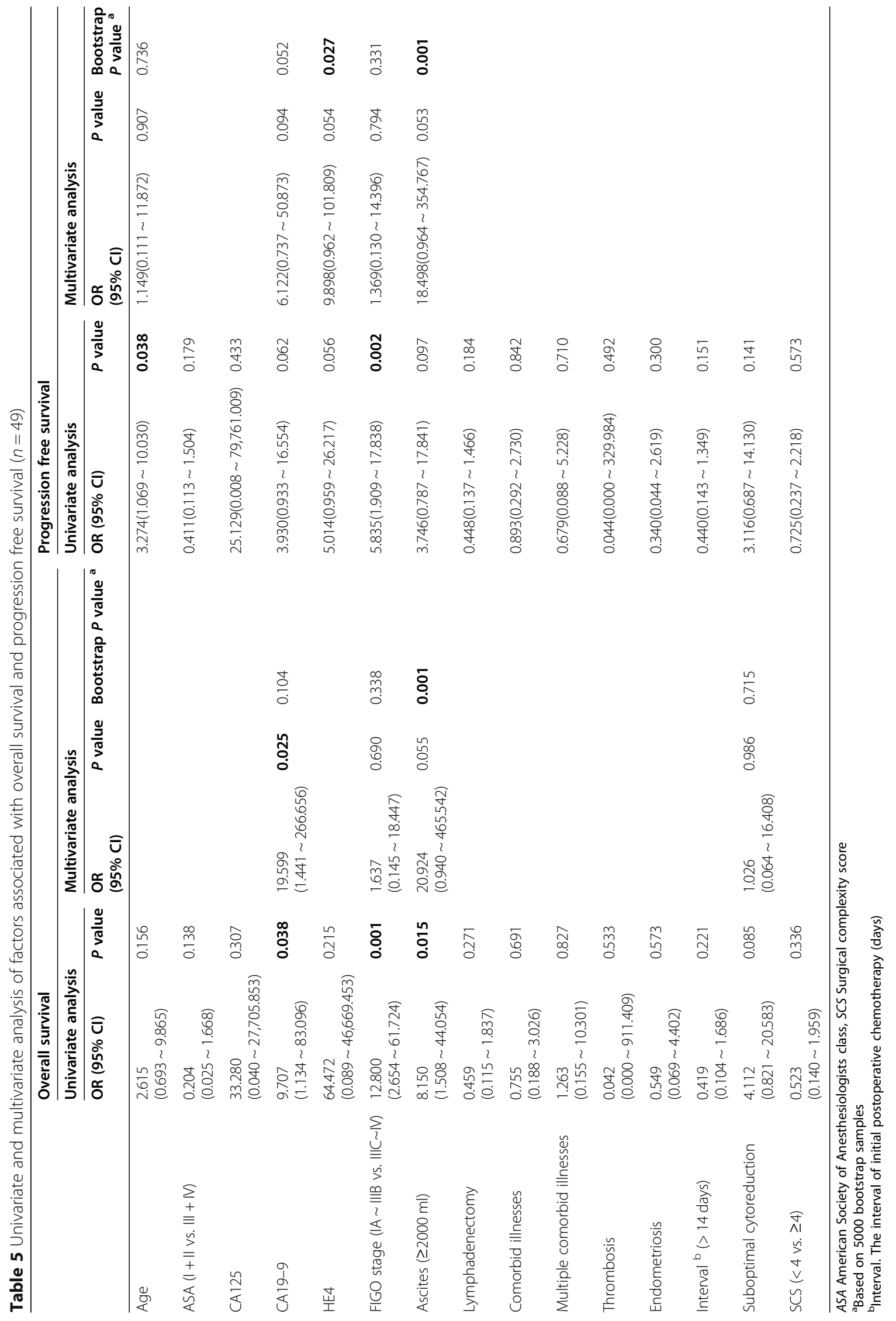



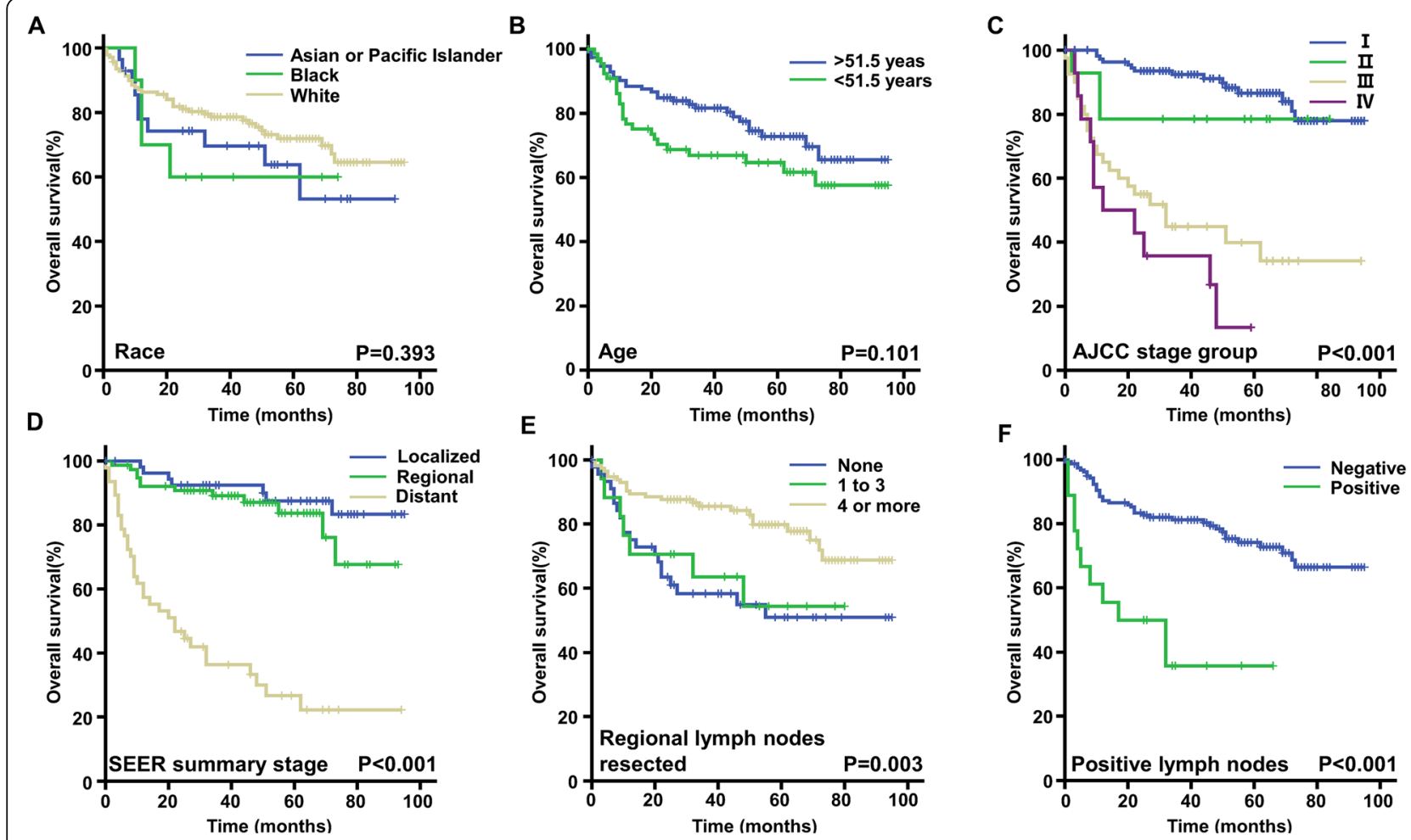

Fig. 2 Kaplan-Meier curves for overall survival of OCCC patients obtained from SEER database. Grouped by race (a), age (b)), AJCC stage group (c), SEER Summary stage $(\mathbf{d})$, the number of regional lymph nodes resected (e), positive lymph nodes (f) for all the 179 patients obtained from SEER database

Table 6 Multivariate analysis of factors associated with overall survival of the data from SEER database $(n=179)$

\begin{tabular}{|c|c|c|c|}
\hline & \multicolumn{3}{|l|}{ Multivariate analysis } \\
\hline & OR $(95 \% \mathrm{Cl})$ & $P$ value & $P$ value ${ }^{a}$ \\
\hline \multicolumn{4}{|l|}{ AJCC stage group } \\
\hline । & 1 & & \\
\hline$\|$ & $1.880(0.186 \sim 18.983)$ & 0.593 & 0.354 \\
\hline III & $3.043(0.255 \sim 36.253)$ & 0.378 & 0.214 \\
\hline IV & $0.526(0.236 \sim 1.171)$ & 0.116 & 0.119 \\
\hline \multicolumn{4}{|l|}{ Summary stage } \\
\hline Localized & 1 & & \\
\hline Regional & $1.361(0.489 \sim 3.787)$ & 0.555 & 0.556 \\
\hline Distant & $21.152(2.019 \sim 221.646)$ & 0.011 & 0.009 \\
\hline \multicolumn{4}{|c|}{ Regional lymph nodes removed } \\
\hline None & 1 & & \\
\hline 1 to 3 regions & $1.847(0.929 \sim 3.672)$ & 0.080 & 0.103 \\
\hline 4 or more regions & $0.621(0.243 \sim 1.587)$ & 0.319 & 0.370 \\
\hline Positive lymph nodes & $5.531(2.102 \sim 14.553)$ & 0.001 & 0.002 \\
\hline
\end{tabular}

Based on 5000 bootstrap samples endometriosis, however, this difference was not significant $(P=0.078)$. Unfortunately, due to the small sample of our research, survival analysis of OCCC patients with and without endometriosis could not be constructed.

Currently, there is no specific biomarker for OCCC, patients with OCCC usually present with a mild elevation of serum CA125 [9]. In the present study, $19.77 \%$ of the patients had a normal level of CA125, and $38.37 \%$ of the patients had a CA125 level of $<100 \mathrm{U} / \mathrm{mL}$. Thus, there is a need to identify the novel diagnostic markers to improve the early diagnosis of OCCC. Chronic inflammation appears to have an effect on tumorigenesis and response to therapy, as well as affecting prognosis [22]. Several systemic inflammatory response (SIR) biomarkers have been investigated as potential biomarkers of OCCC, such as the neutrophil-to-lymphocyte ratio (NLR) and lymphocyte-to-monocyte ratio (LMR). Most OCCC patients diagnosed at an early stage showed complete response to initial treatment with decreased NLR levels, and NLR was found to increase to preoperative high levels when recurrence, reflecting inflammation caused by the tumor [23]. Low LMR has been shown to be associated with advanced-stage disease, LN metastases, platinum-resistance, and poor prognosis, suggesting a decreased level of peripheral lymphocytes results in weakened immune surveillance and poor response to 
chemotherapy [22]. In addition, several potential diagnostic novel biomarkers were gradually discovered and used for OCCC, such as HNF1 $\beta$, which is expressed in almost all cases of OCCC and now is used to distinguish histological subtypes by immunohistochemistry (IHC) [24]. OCCC lesions tend to be positive for CK7 and negative for CK20, ER, PR, WT-1, and p53 [10, 25]. Testing negative for $\alpha$-fetoprotein and CD10 can be used to exclude yolk cell tumors and renal cell carcinoma [26]. Since there are few reports about combining those biomarkers, we analyzed immunohistochemical results of our patients, and $55 \%(11 / 20)$ of them had positive HNF1 $\beta$ and negative WT-1, ER, and PR. However, there remains an urgent need to discover novel biomarkers in peripheral blood or body fluids and validate their efficacy in the diagnosis of OCCC.

VTE, containing deep vein thrombosis (DVT) and pulmonary embolism (PE), are common in patients with OCCC, with a 2.5-4 times higher risk than in other subtypes of EOC [20, 27]. VTE is more commonly seen in advanced stage OCCC (21.9\%) compared with earlystage $(8.2 \%)$ disease, and occurred most commonly prior to primary surgery (36.4\%), or with recurrence or progression (33.3\%) [20]. There are some measurable biomarkers of increased risk of VTE in ovarian cancer, such as elevated platelet count, white blood cell counts, ddimer and CA125 level, decreased hemoglobin and albumin levels preoperatively, and elevated d-dimer and decreased albumin postoperatively [28]. In our study, 8 patients developed VTE (Table 2), 4 preoperatively and 4 postoperatively. The low occurrence of VTE in our research might be associated with the use of appropriate prophylaxis. In spite of prophylaxis, patients with OCCC can still develop VTE, suggesting that an aggressive postoperative anticoagulation regimen and prolonged post-discharge VTE prophylaxis should be considered for patients with OCCC.

Staging surgery or optimal cytoreduction combined with chemotherapy is a common therapeutic strategy recommended for OCCC, however, only $11-27 \%$ of patients with OCCC respond to conventional platinumbased chemotherapy, resulting in a poor prognosis [29, 30]. Disorder of the cell's detoxification effects of the glutathione system, low proliferation activity of OCCC, and overexpression of EGFR, HNF1 $\beta$, and HER2 may be involved in chemoresistance [31]. In our clinical practice, OCCC patients often developed resistance characterized by a slow decline or elevation of tumor markers during postoperative chemotherapy. However, in the absence of more effective treatments, platinum-based chemotherapy remains the first-line adjuvant therapy for OCCC patients, and more effective therapies are urgently needed. Recently, several targeted therapies and immunotherapies have been investigated for use in OCCC, such as
PARP, EZH2, and ATR inhibitors combined with synthetic lethality of ARID1A-deficiency, and MAPK/PI3K/ HER2, VEGF/bFGF/PDGF, HNF1 $\beta$, and PD-1/PD-L1 inhibitors. Some regimens have demonstrated efficacy and revealed a potential therapeutic benefit for OCCC patients, but further research is required [32-34]. We have collected all cases diagnosed with OCCC, and found that almost all of them were received surgery firstly, only one patient received NACT prior to debulking and she is still alive, this patient was not included in our data. We suspect the low number of NACT in patients with OCCC may be the cause of the following point: OCCC patients tend to be younger when they were firstly diagnosed without so many complications as the elders, which means they can withstand the extensive optimal cytoreduction. The same situation was also found in two clinical trials, CHORUS and EORTC55971 which compared the outcomes of patients with advanced EOC who had primary cytoreduction and NACT (neoadjuvant chemotherapy) + interval cytoreduction, and the incidence of OCCC was low (1.5 to 6.0\%) in both trials [35, 36]. Therefore, the importance of NACT for OCCC requires multi-center and large-sample clinical studies.

Survival analysis of our clinical data identified levels of CA19-9 and ascites $(\geq 2000 \mathrm{ml})$ as independent OS related factors in OCCC; HE4 and ascites $(\geq 2000 \mathrm{ml})$, as independent PFS related factors in OCCC, have rarely been reported in previous studies. Elevated postoperative CA19-9 has been reported as an independent risk factor for reduced survival outcomes in OCCC patients with normal postoperative CA125 levels [37]. HE4 is not commonly used in OCCC prediction, however, Mckinnon found that since HE4 is sensitive to hormonal treatment and menstrual cycle variation, it may be potentially superior to CA125 as an endometriosis marker and therefore has potential as a marker for the risk of developing ovarian cancer [38]. Suboptimal cytoreduction and advanced stage have been found to be associated with less favorable survival outcomes in univariate analysis, however, neither is an independent prognostic factor in the present study, which may be related to the small sample size. The extent of lymph node dissection in patients with early OCCC has always been a controversial topic. In our survival analysis of 179 cases of OCCC from the SEER, we found that positive $\mathrm{LN}$ is an independent prognostic factor. OCCC tends to metastasize most frequently via the lymphatic system [39]. However, among patients in our hospital, only $4.48 \%$ of patients had metastatic LNs, which may be due to the fact that most $(89.55 \%)$ of the 67 patients who received lymphadenectomy were at an early stage. Mueller found that $4.4-20 \%$ of clinically stage I OCCC had lymph node involvement, and this rate will be higher with positive cytology or ovarian surface involvement, 
accounting for $37.5 \%$ of metastases [40]. Therefore, we suggested that systematic pelvic and para-aortic lymphadenectomy is vital to accurately define the stage, provide prognostic information, and guide adjuvant therapy. Since ascites were mostly in advanced- stage disease, and cytoreduction was only used for advanced-stage of OCCC, we have also conducted a stratified multivariate analysis in early-stage and advanced-stage disease, respectively. However, according to the results presented in sTable5 and sTable6, CA19-9, HE4, and multiple comorbid illnesses are related to OS of patients with OCCC at early stage; lymphadenectomy and SCS $\geq 4$ are related to OS of patients with OCCC at advanced stage in univariate analysis respectively, while only a high ASA score is a significant indicator for a poor PFS of patients with advanced OCCC in multivariate analysis.

However, this is a retrospective research in a singlecenter, our study still has some limitations. Due to the small sample of our study, we also analyzed the data from 179 patients enrolled from the SEER database, which used the different stage systems, including SEER summary stages as well as AJCC stage systems. Similar to us, most patients in this database were diagnosed at early stage $(77.65 \%)$. In our study, there are only $3(4.48 \%)$ patients were found to have positive $\mathrm{LN}$, we cannot know the statistical relationship between positive lymph nodes and prognosis. However, in the SEER database, lymphadenectomy was performed in 134 patients. $14.18 \%$ patients had positive $\mathrm{LN}$, and positive LN was significantly associated with OS, which has made up for the limitations of our research. However, we still cannot find the most of the key findings of this study such as the HE4 and CA19-9 which were not available in SEER data. Therefore, multi-center clinical research with large samples is very necessary to investigate this rare disease.

In conclusion, our study presents the clinicopathological features, treatment regimens, and prognosis of OCCC in China, and confirms that OCCC typically presents at an early stage and at a younger age, with a mild elevation in CA125 level. Positive HNF1 $\beta$, and negative WT-1, ER, and PR are reliable immunohistochemical indicators of OCCC. Patients with early-stage OCCC tend to have a better OS and PFS, and CA19-9, HE4, massive ascites, and positive lymph nodes are independent prognostic indicators. The present study confirms the unique features of OCCC, and further research is required to illustrate the molecular mechanisms, discover novel diagnostic biomarkers and targeted therapies, in order to contribute to the early diagnosis and better prognosis of OCCC.

\section{Abbreviations}

EOC: Epithelial ovarian carcinoma; OCCC: Ovarian clear cell carcinoma; SEER: Surveillance, Epidemiology and End Results; VTE: Venous thromboembolism; OS: Overall survival; PFS: Progression-free survival;
HGSC: High-grade serous carcinoma; HNF1 $\beta$ : hepatocyte nuclear factor $1 \beta$; ER: Estrogen receptor; PR: Progesterone receptor; WT-1: Wilms Tumor 1: PIK3CA: Phosphatidylinositol-4,5-bisphosphate 3-kinase catalytic subunit alpha; ARID1A: AT-rich interactive domain-containing protein 1A; LN: Lymph node; USTC: The First Affiliated Hospital of University of Science and Technology of China; CT: Computed tomography; MRI: Magnetic resonance imaging; PET-CT: Positron emission tomography-CT; BMI: Body mass index; ASA: American Society of Anesthesiologists class; SCS: Surgical complexity score; ELICA: Electrochemiluminescence immunoassay; FIGO: International Federation of Gynecology and Obstetrics; WHO: World Health Organization; AJCC: American Joint Committee on Cancer; ROC: Receiver operating characteristic; SIR: Systemic inflammatory response; NLR: Neutrophil-tolymphocyte ratio; LMR: Lymphocyte-to-monocyte ratio;

IHC: Immunohistochemistry; DVT: Deep vein thrombosis; PE: Pulmonary embolism

\section{Supplementary Information}

The online version contains supplementary material available at https://doi. org/10.1186/s12885-021-08061-7.

Additional file 1: sTable1. Clinical characteristics of patients with/ without endometriosis. a. Mean \pm standard deviation, range; NA. Not available. sTable2. The treatment and survival outcomes of the 14 patients with OCCC who had a recurrence. sTable3. The prognostic outcomes of patients with OCCC at the stage of IC. sTable4. The comparison of survival time between patients with OCCC at stage IC1 and IC2/3. sTable5. Multivariate analysis of factors associated with overall survival among patients at early stage $(n=37)$. a. Based on 5000 bootstrap samples. b. Interval. The interval of initial postoperative chemotherapy (days). ASA: American Society of Anesthesiologists class; SCS: Surgical complexity score. sTable6. Multivariate analysis of factors associated with overall survival among patients at advanced stage $(n=$ 12). a. Based on 5000 bootstrap samples. b. Interval. The interval of initial postoperative chemotherapy (days). ASA: American Society of Anesthesiologists class; SCS: Surgical complexity score. sTable7. Power calculation and multiple hypothesis correction of the analysis of overall survival among the 49 patients with OCCC at early and advanced stage. sTable8. Power calculation and multiple hypothesis correction of the analysis of progression free survival among the 49 patients with OCCC at early and advanced stage.

\section{Acknowledgements}

Not Applicable.

\section{Authors' contributions}

CZ was a major contributor in designing study and writing the manuscript; $\mathrm{JZ}$ analyzed and interpreted the patient data; LQ and HL collected the patient data; ZS, DW and WZ completed the patient's follow-up together; $W X$ and $Y Z$ play an important role in critical revision of the manuscript. All authors read and approved the final manuscript.

\section{Funding}

This work was supported by the National Natural Science Foundation of China (81872110, 81902632), National Key Research and Development Program (2018YFC1003900), the Strategic Priority Research Program of the Chinese Academy of Sciences (XDB29030000), the Ministry of Science and Technology of China (2016YFC1303503). The funders had no role in the study design, data collection and analysis, decision to publish, or preparation of the manuscript.

\section{Availability of data and materials}

The datasets used and/or analyzed during the current study are available from the corresponding author on reasonable request.

\section{Declarations}

Ethics approval and consent to participate

The study was ethically reviewed and approved by the ethics committees of The First Affiliated Hospital of USTC. Since this study does not involve the 
collection of human tissues, blood and other samples, only the original medical records would be collected for retrospective analysis, we obtained verbal informed consent from all participants, and the ethics committee approved this procedure.

\section{Consent for publication}

Not Applicable.

\section{Competing interests}

No conflicts of interest to disclose.

\section{Author details}

${ }^{1}$ Department of Obstetrics and Gynecology, Anhui Provincial Hospital, Anhui Medical University, Hefei 230001, China. ${ }^{2}$ Department of Obstetrics and Gynecology, The First Affiliated Hospital of USTC, Division of Life Sciences and Medicine, University of Science and Technology of China, Hefei 230001, Anhui, China. ${ }^{3}$ Division of Molecular Medicine, Hefei National Laboratory for Physical Sciences at Microscale, The CAS Key Laboratory of Innate Immunity and Chronic Disease, School of Life Sciences, University of Science and Technology of China, Hefei, China. Institute of Immunology, University of Science and Technology of China, Hefei, China.

\section{Received: 21 September 2020 Accepted: 18 March 2021}

Published online: 25 March 2021

\section{References}

1. Siegel RL, Miller KD, Jemal A. Cancer statistics, 2020. CA Cancer J Clin. 2020; 70(1):7-30. https://doi.org/10.3322/caac.21590.

2. Reid BM, Permuth JB, Sellers TA. Epidemiology of ovarian cancer: a review. Cancer Biol Med. 2017;14(1):9-32. https://doi.org/10.20892/j.issn.2095-3941.2 016.0084 .

3. Oliver KE, Brady WE, Birrer M, Gershenson DM, Fleming G, Copeland LJ, et al. An evaluation of progression free survival and overall survival of ovarian cancer patients with clear cell carcinoma versus serous carcinoma treated with platinum therapy: an NRG oncology/gynecologic oncology group experience. Gynecol Oncol. 2017;147(2):243-9. https://doi.org/10.1016/j. ygyno.2017.08.004

4. Kim SI, Lim MC, Lim J, Won YJ, Seo SS, Kang S, et al. Incidence of epithelial ovarian cancer according to histologic subtypes in Korea, 1999 to 2012. J Gynecol Oncol. 2016;27(1):e5. https://doi.org/10.3802/jgo.2016.27.e5.

5. Okamoto A, Glasspool RM, Mabuchi S, Matsumura N, Nomura H, Itamochi H, et al. Gynecologic Cancer InterGroup (GCIG) consensus review for clear cell carcinoma of the ovary. Int J Gynecol Cancer. 2014;24(9 Suppl 3):S20-5. https://doi.org/10.1097/IGC.0000000000000289.

6. Yamamoto A, Johnstone EB, Bloom MS, Huddleston HG, Fujimoto WY. A higher prevalence of endometriosis among Asian women does not contribute to poorer IVF outcomes. J Assist Reprod Genet. 2017;34(6):76574. https://doi.org/10.1007/s10815-017-0919-1.

7. Pearce CL, Templeman C, Rossing MA, Lee A, Near AM, Webb PM, et al. Association between endometriosis and risk of histological subtypes of ovarian cancer: a pooled analysis of case-control studies. Lancet Oncol. 2012;13(4):385-94. https://doi.org/10.1016/S1470-2045(11)70404-1.

8. Machida H, Matsuo K, Yamagami W, Ebina Y, Kobayashi Y, Tabata T, et al. Trends and characteristics of epithelial ovarian cancer in Japan between 2002 and 2015: a JSGO-JSOG joint study. Gynecol Oncol. 2019;153(3):58996. https://doi.org/10.1016/j.ygyno.2019.03.243.

9. Liu H, Xu Y, Ji J, Dong R, Qiu H, Dai X. Prognosis of ovarian clear cell cancer compared with other epithelial cancer types: a population-based analysis. Oncol Lett. 2020;19(3):1947-57. https://doi.org/10.3892/ol.2020.11252.

10. Prat J. Ovarian carcinomas: five distinct diseases with different origins, genetic alterations, and clinicopathological features. Virchows Arch. 2012; 460(3):237-49. https://doi.org/10.1007/s00428-012-1203-5.

11. Rice MS, Hankinson SE, Tworoger SS. Tubal ligation, hysterectomy, unilateral oophorectomy, and risk of ovarian cancer in the Nurses' health studies. Fertil Steril. 2014;102(1):192-8 e3. https://doi.org/10.1016/j.fertnstert.2014.03. 041.

12. Enomoto T, Aoki D, Hattori $K$, Jinushi M, Sugiyama T. The first Japanese nationwide multicenter study of BRCA mutation testing in ovarian cancer: CHARacterizing the cross-sectionaL approach to ovarian cancer geneTic TEsting of BRCA (CHARLOTTE). Int J Gynecol Cancer. 2019;29(6):1043-9. https://doi.org/10.1136/ijgc-2019-000384.
13. Tang $H$, Liu $Y$, Wang $X$, Guan $L$, Chen $W$, Jiang $H$, et al. Clear cell carcinoma of the ovary: Clinicopathologic features and outcomes in a Chinese cohort. Medicine (Baltimore). 2018;97(21):e10881. https://doi.org/10.1097/MD. 0000000000010881.

14. Lee HY, Hong JH, Byun JH, Kim HJ, Baek SK, Kim JY, et al. Clinical characteristics of clear cell ovarian Cancer: a retrospective multicenter experience of 308 patients in South Korea. Cancer Res Treat. 2020;52(1):27783. https://doi.org/10.4143/crt.2019.292.

15. Takahashi K, Takenaka M, Kawabata A, Yanaihara N, Okamoto A. Rethinking of treatment strategies and clinical management in ovarian clear cell carcinoma. Int J Clin Oncol. 2020;25(3):425-31. https://doi.org/10.1007/s1014 7-020-01625-w.

16. King CM, Barbara C, Prentice A, Brenton JD, Charnock-Jones DS. Models of endometriosis and their utility in studying progression to ovarian clear cell carcinoma. J Pathol. 2016;238(2):185-96. https://doi.org/10.1002/path.4657.

17. Ishibashi H, Takano M, Miyamoto M, Soyama H, Matsuura H, Aoyama T, et al. Role of endometriosis as a prognostic factor for post-progression survival in ovarian clear cell carcinoma. Mol Clin Oncol. 2017;7(6):1027-31. https://doi. org/10.3892/mco.2017.1468.

18. Paik ES, Kim T-J, Choi CH, Kim B-G, Bae D-S, Lee J-W. Clinical outcomes of patients with clear cell and endometrioid ovarian cancer arising from endometriosis. J Gynecol Oncol. 2018;29(2):e18.

19. Son JH, Yoon S, Kim S, Kong TW, Paek J, Chang SJ, et al. Clinicopathologic characteristics of ovarian clear cell carcinoma in the background of endometrioma: a surveillance strategy for an early detection of malignant transformation in patients with asymptomatic endometrioma. Obstet Gynecol Sci. 2019;62(1):27-34. https://doi.org/10.5468/ogs.2019.62.1.27.

20. Ye S, Yang J, Cao D, Bai H, Huang $\mathrm{H}, \mathrm{Wu}$ M, et al. Characteristic and prognostic implication of venous thromboembolism in ovarian clear cell carcinoma: a 12-year retrospective study. PLoS One. 2015;10(3):e0121818. https://doi.org/10.1371/journal.pone.0121818.

21. Shuang $Y$, Yang J, Yan $Y$, et al. Comparative study of ovarian clear cell carcinoma with and without endometriosis in People's Republic of China. Fertil Steril. 2014;102(6):1656-62.

22. Kwon BS, Jeong DH, Byun JM, Lee TH, Choi KU, Song YJ, et al. Prognostic value of preoperative lymphocyte-monocyte ratio in patients with ovarian clear cell carcinoma. J Cancer. 2018;9(7):1127-34. https://doi.org/10.7150/ jca.24057.

23. Yoshida K, Yoshikawa N, Shirakawa A, Niimi K, Suzuki S, Kajiyama H, et al. Prognostic value of neutrophil-to-lymphocyte ratio in early-stage ovarian clear-cell carcinoma. J Gynecol Oncol. 2019;30(6):e85. https://doi.org/10.3 802/jgo.2019.30.e85.

24. Mabuchi S, Sugiyama T, Kimura T. Clear cell carcinoma of the ovary: molecular insights and future therapeutic perspectives. J Gynecol Oncol. 2016;27(3):e31. https://doi.org/10.3802/jgo.2016.27.e31.

25. Köbel M, Piskorz AM, Lee S, Lui S, Brenton JD. Optimized p53 immunohistochemistry is an accurate predictor of TP53 mutation in ovarian carcinoma. J Pathol Clin Res. 2016;2(4):247-58. https://doi.org/10.1002/cjp2.53.

26. Marks El, Brown VS, Dizon DS. Genomic and molecular abnormalities in gynecologic clear cell carcinoma. Am J Clin Oncol. 2020;43(2):139-45. https://doi.org/10.1097/COC.0000000000000641.

27. Swier $\mathrm{N}$, Versteeg HH. Reciprocal links between venous thromboembolism, coagulation factors and ovarian cancer progression. Thromb Res. 2017;150: 8-18. https://doi.org/10.1016/j.thromres.2016.12.002.

28. Zhou Q, Zhu C, Shen Z, Zhang T, Li M, Zhu J, et al. Incidence and potential predictors of thromboembolic events in epithelial ovarian carcinoma patients during perioperative period. Eur J Surg Oncol. 2020;46(5):855-61. https://doi.org/10.1016/j.ejso.2020.01.026.

29. Takano M, Tsuda H, Sugiyama T. Clear cell carcinoma of the ovary: is there a role of histology-specific treatment? J Exp Clin Cancer Res. 2012;31(1):53. https://doi.org/10.1186/1756-9966-31-53.

30. Kobayashi H, Sugimoto H, Onishi S, Nakano K. Novel biomarker candidates for the diagnosis of ovarian clear cell carcinoma. Oncol Lett. 2015;10(2):6128. https://doi.org/10.3892/ol.2015.3367.

31. Itamochi $\mathrm{H}$, Kigawa J, Terakawa N. Mechanisms of chemoresistance and poor prognosis in ovarian clear cell carcinoma. Cancer Sci. 2008;99(4):653-8. https://doi.org/10.1111/j.1349-7006.2008.00747.x.

32. Caumanns JJ, Wisman GBA, Berns K, van der Zee AGJ, de Jong S. ARID1A mutant ovarian clear cell carcinoma: a clear target for synthetic lethal strategies. Biochim Biophys Acta Rev Cancer. 2018;1870(2):176-84. https:// doi.org/10.1016/j.bbcan.2018.07.005. 
33. Konstantinopoulos PA, Brady WE, Farley J, Armstrong A, Uyar DS, Gershenson DM. Phase II study of single-agent cabozantinib in patients with recurrent clear cell ovarian, primary peritoneal or fallopian tube cancer (NRG-GY001). Gynecol Oncol. 2018;150(1):9-13. https://doi.org/10.1016/j. ygyno.2018.04.572.

34. Komiyama S, Kato K, Inokuchi Y, Takano H, Matsumoto T, Hongo A, et al. Bevacizumab combined with platinum-taxane chemotherapy as first-line treatment for advanced ovarian cancer: a prospective observational study of safety and efficacy in Japanese patients (JGOG3022 trial). Int J Clin Oncol. 2019;24(1):103-14. https://doi.org/10.1007/s10147-018-1319-y.

35. Vergote I, Trope CG, Amant F, Kristensen GB, Ehlen T, Johnson N, et al. Neoadjuvant chemotherapy or primary surgery in stage IIIC or IV ovarian cancer. N Engl J Med. 2010;363(10):943-53. https://doi.org/10.1056/NEJMoa 0908806.

36. Kehoe S, Hook J, Nankivell M, Jayson GC, Kitchener H, Lopes T, et al. Primary chemotherapy versus primary surgery for newly diagnosed advanced ovarian cancer (CHORUS): an open-label, randomised, controlled, noninferiority trial. Lancet. 2015;386(9990):249-57. https://doi.org/10.1016/S01406736(14)62223-6.

37. Zhu J, Jiang L, Wen H, Bi R, Wu X, Ju X. Prognostic value of serum CA19-9 and perioperative CA-125 levels in ovarian clear cell carcinoma. Int J Gynecol Cancer. 2018;28(6):1108-16. https://doi.org/10.1097//GC 0000000000001293.

38. McKinnon B, Mueller MD, Nirgianakis K, Bersinger NA. Comparison of ovarian cancer markers in endometriosis favours HE4 over CA125. Mol Med Rep. 2015:12(4):5179-84. https://doi.org/10.3892/mmr.2015.4062.

39. Szubert M, Suzin J, Obirek K, Sochacka A, Loszakiewicz M. Clear cell ovarian cancer and endometriosis: is there a relationship? Prz Menopauzalny. 2016; 15(2):85-9. https://doi.org/10.5114/pm.2016.61190.

40. Mueller JJ, Holzapfel M, Han CH, Santos K, Gunderson C, Moore K, et al. Staging lymphadenectomy in patients with clear cell carcinoma of the ovary. Int J Gynecol Cancer. 2016;26(1):120-4. https://doi.org/10.1097/IGC. 0000000000000559 .

\section{Publisher's Note}

Springer Nature remains neutral with regard to jurisdictional claims in published maps and institutional affiliations.

Ready to submit your research? Choose BMC and benefit from:

- fast, convenient online submission

- thorough peer review by experienced researchers in your field

- rapid publication on acceptance

- support for research data, including large and complex data types

- gold Open Access which fosters wider collaboration and increased citations

- maximum visibility for your research: over $100 \mathrm{M}$ website views per year

At $\mathrm{BMC}$, research is always in progress.

Learn more biomedcentral.com/submissions 